\title{
Artículo Original: VALIDACIÓN DE LA MEDIDA DE INDEPENDENCIA PARA LA MÉDULA ESPINAL VERSIÓN III EN ESPAÑOL (eSCIM III), EN PACIENTES HOSPITALIZADOS EN LA UNIDAD DE LESIONES MEDULARES DEL CENTRO NACIONAL DE REHABILITACIÓN EN LOS MESES DE ENERO, FEBRERO Y ABRIL DEL 2015
}

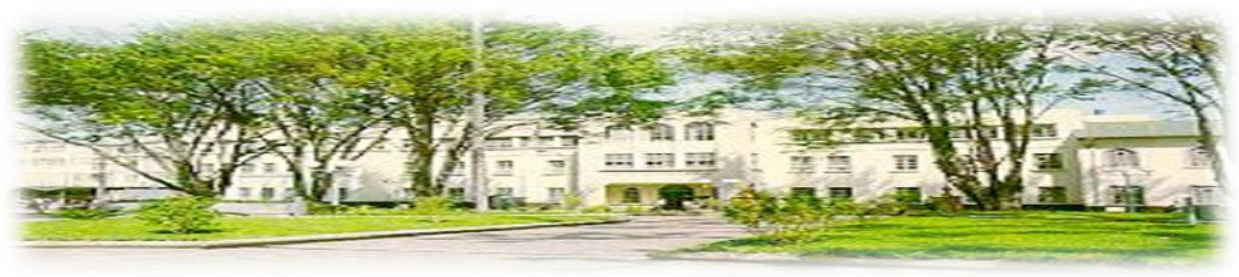

Hospital San Juan de Dios, San José, Costa Rica. Fundado en 1845

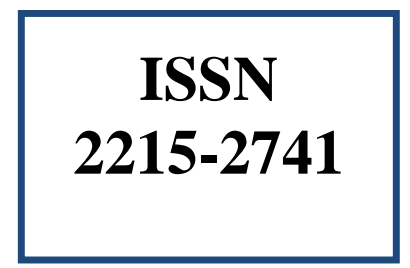

Recibido: 15/03/2016

Aceptado: 06/10/2016

\author{
María Félix Sánchez Solera ${ }^{1}$ \\ María José Gallardo ${ }^{2}$ \\ Sirlene González ${ }^{3}$
}

\begin{abstract}
${ }^{1}$ Médico Asistente, Especialista en Medicina Física y Rehabilitación en el Centro Nacional de Rehabilitación de la Caja Costarricense de Seguro Social. Correo electrónico: marife.sanchez@gmail.com

${ }^{2}$ Médico Asistente, Especialista en medicina Física y Rehabilitación en el Centro Nacional de Rehabilitación de la Caja Costarricense de Seguro Social. Correo electrónico:

${ }^{3}$ Médico Residente de tercer año Medicina Física y Rehabilitación en el Centro Nacional de Rehabilitación de la Caja Costarricense de Seguro Social. Correo electrónico:
\end{abstract}

\section{RESUMEN}

La Medida de Independencia de la Médula Espinal fue propuesta en 1997 por los médicos Catz, Itzkovich, Agranov del Departamento de Lesiones Medulares del Hospital de Rehabilitación Loewenstein, Israel; como una escala de discapacidad innovadora, diseñada específicamente para personas con lesiones medulares. La SCIM es la única escala que mide independencia en todos los aspectos de las actividades de vida diaria relevantes para los pacientes con lesiones medulares. Los valores relativos de cada componente de la SCIM fueron basados en la combinación de aspectos tales como, dificultad preestablecida y tiempo requerido para llevar a cabo la actividad. . Estos pesos relativos fueron determinados basados en un consenso de profesionales de Israel y modificado en la SCIM III con las consultas realizadas con expertos americanos y europeos. El motivo principal para el desarrollo de la SCIM fue la necesidad de medir y monitorear la efectividad de la rehabilitación de pacientes con lesión medular de una manera precisa. Objetivo: Disponer de una herramienta válida, reproducible y con alta coherencia interna para ser aplicada en pacientes con 
lesiones medulares hospitalizados en el Centro Nacional de Rehabilitación en los meses de enero, febrero y abril del 2015. Método: Estudio descriptivo, transversal, observacional de 49 pacientes mayores de edad, hospitalizados en la unidad de lesiones medulares del Centro Nacional de Rehabilitación. Se recogieron datos sociodemográficos y clínicos básicos. Dos evaluadores evaluaron a los pacientes utilizando la versión en español de la SCIM III. Se estudiaron los descriptivos de los parámetros recogidos y las características psicométricas del cuestionario (confiabilidad y validez). Resultados: La distribución fue de $76 \%$ hombres y $24 \%$ mujeres, El déficit neurológico encontrado en los pacientes es $40,8 \%$ tetraplejía incompleta, $30,6 \%$ paraplejía incompleta, 26,5\% paraplejía completa y $2 \%$ tetraplejía completa. Se obtuvo un valor del Alpha de Cronbach de 0,919 para el evaluador 1, 0,918 para el evaluador 2 y 0,920 para la evaluación al egreso. Ambos observadores tuvieron una concordancia muy buena, incluso en movilidad dormitorio y baño la concordancia fue absoluta con un índice de Kappa entre 0,81 y 1. Conclusión: la Medida de Independencia de la Médula Espinal versión III en español, es una herramienta válida y confiable para la evaluación funcional de los pacientes con lesiones medulares del Centro Nacional de Rehabilitación.

\section{PALABRAS CLAVE}

Validación, eSCIM III, evaluación funcional.

\section{ABSTRACT}

The Spinal Cord Independence Measure (SCIM) was proposed by the doctors Catz, Itzkovich and Agranov, of the Spinal Cord Injury Department of the Hospital of Rehabilitation of Loewenstein Israel, as an innovative scale for disability, designed specifically for people with spinal cord injury. The SCIM is the only scale that measures independence in every aspect of daily activities relevant for patients with spinal cord injury. The relative values of each component of the SCIM were based on the combination of matters such as preestablished difficulty and required time to perform the activity. These relative weights were determined based on a consensus of Israel professionals and modified on the SCIM III with the consultations made with American and European experts. The principal motive for the development of the SCIM was the necessity to measure and monitor the effectiveness of the rehabilitation of patients with spinal cord injury in a precise way. Objective: To dispose of a valid, reproducible and with high internal coherence tool to be applied in patients with spinal cord injury hospitalized in the National Centre of Rehabilitation during the months of January, February and April 2015. Method: Descriptive, transversal study of 49 adult patients, hospitalized in the unity of spinal cord injury of the National Centre of Rehabilitation. The sociodemographic and basic clinical data were recorded. Two evaluators assessed the patients using the spanish version of the SCIM III. The descriptions of the collected parameters and the psychometric characteristics of the questionnaire (reliability and validity) were studied. Results: The distribution was of $76 \%$ men and $24 \%$ women. The neurological deficit found in the patients was 40,8\% incomplete quadriplegia, 30,6\% incomplete paraplegia, $26,5 \%$ complete paraplegia and $2 \%$ complete quadriplegia. We obtained an Alpha of Cronbach value of 0,919 for evaluator 1 , of 0,918 for evaluator 2 and of 0,920 at the discharge evaluation. Both observers had a very good consistency, even in mobility in the bedroom and bathroom the consistency is absolute, with a Kappa index between 0,81 and 1. Conclusion: The Spinal Cord Independence Measure, III version in spanish, is a valid and reliable tool for the functional assessment of the patients with spinal cord injury of the National Centre of Rehabilitation.

\section{KEY WORDS}

Validation, eSCIM III, functional evaluation

\section{INTRODUCCIÓN}

La lesión de la médula espinal (LME), es el daño que se produce en cualquier sitio de esta estructura del Sistema Nervioso Central, la cual se extiende desde el foramen magno hasta la cauda equina. La LME se define como un proceso de etiología variable que resulta de la alteración temporal o permanente de la función motora, sensitiva y/o autonómica. La lesión que sufre la médula espinal, conlleva a un déficit neurológico con efectos a

Rev Cl EMed UCR www.revistaclinicahsjd.ucr.ac.cr abril 2016 
largo plazo que persisten a lo largo de la vida. Todas estas alteraciones habitualmente se presentan por debajo del nivel de la lesión. ${ }^{1}$

Las condiciones asociadas lesiones medulares representan un importante problema de salud, debido a su complejidad clínica, requerimiento de hospitalizaciones prolongadas y necesidad de seguimientos clínicos debido a la alta frecuencia de complicaciones. ${ }^{2}$ La meta principal en la rehabilitación de las personas con lesión medular es maximizar la restauración de la funcionalidad física. Es imperativo documentar la extensión de la recuperación para: evaluar la eficacia del tratamiento, determinar el costo-beneficio de las intervenciones, evaluar la calidad del cuidado dado al paciente y brindar información de pronóstico. ${ }^{2,3}$

La Medida de Independencia de la Médula Espinal, es la única escala de funcionalidad diseñada específicamente para pacientes con lesiones medulares, es fácil de usar y no requiere manual, ya que los criterios del puntaje son detallados en la escala. . ${ }^{3}$

La SCIM fue diseñada específicamente para cuantificar la evaluación funcional de los pacientes con lesión de la médula espinal. Desde su creación, diferentes versiones han sido desarrolladas, lo que resulta en que cada versión es una herramienta más precisa y sensible en comparación con la anterior. $^{3}$

Consta de 3 subescalas que cubren los subconjuntos relacionados, pero distintos, del autocuidado (6 artículos; rango de puntuación, 0-20); la respiración y manejo de esfínteres (4 artículos; rango de puntuación, 0-40); y la movilidad (9 artículos; rango de puntuación, 0-40). El subconjunto movilidad se subdivide en 2 subescalas: habitación y aseo, tanto en interiores como en exteriores. La puntuación total varía de 0 a 100 , donde 0 puntos corresponden al mayor grado de dependencia. ${ }^{3}$

La importancia de disponer de instrumentos validados en el ámbito de la salud y en el idioma original, es fundamental para poder comparar los resultados obtenidos con otros estudios que hayan utilizado el mismo instrumento. ${ }^{1,4} \mathrm{El}$ análisis de las características métricas del instrumento es un proceso complejo que implica la evaluación de la confiabilidad y validez del constructo.
Las dos características métricas esenciales para valorar la precisión de un instrumento son la confiabilidad y la validez. La primera se define como la propiedad que designa la constancia y precisión de los resultados que obtiene un instrumento al aplicarlo en distintas ocasiones. El concepto no involucra que una confiabilidad alta sea sinónimo de calidad, esto debido a que la validez es lo más importante. Otra de las características es la consistencia interna, esta hace referencia a la consistencia de una medición, es decir si la escala o el test funciona de manera similar bajo diferentes condiciones dependientes del mismo instrumento, del tiempo de aplicación, del que hace la medición, de los sujetos, de interacción entre estas fuentes y del error aleatorio puro. ${ }^{4,5}$

El alfa de Cronbach; se considera el indicador más idóneo porque da un único valor de consistencia y proporciona los datos de la técnica de la fiabilidad mitad y mitad. El rango del alfa de Cronbach oscila de 0 a 1 .

La validez explora el grado del instrumento con el que mide lo que debería medir, es decir aquello para lo que ha sido diseñado. Existen diferentes formas para determinar la validez y cada una de ellas proporciona evidencias a la validación global del instrumento. ${ }^{2,6,7}$

Este estudio pretende averiguar se la SCIM III es una herramienta válida para su uso en los pacientes con lesiones medulares hospitalizados en el Centro Nacional de Rehabilitación.

\section{MATERIALES Y MÉTODOS}

Dos evaluadores aplican la eSCIM III, a 49 pacientes con lesiones medulares al momento del ingreso y del egreso a la Unidad de Lesiones Medulares del Centro Nacional de Rehabilitación. Además se obtienen los puntajes de la Medida de Independencia Funcioanl (MIF) de ingreso y egreso, ya que esta es la escala que se utiliza en la actualidad para la evaluación funcional de estos pacientes. 


\section{RESULTADOS}

La realización de la adaptación cultural se realizó por una especialista filóloga quien recomendó los cambios respectivos de la versión española a una adaptación al castellano para su utilización y exposición a los expertos y pacientes para el programa piloto, orientados a contenido, estructura y uso del lenguaje.

La caracterización de la población se realizó tomando en cuenta el género, edad, etiología de la lesión, déficit neurológico, clasificación ASIA. En la muestra estudiada, se obtuvo que los pacientes lesionados medulares son en su mayoría hombres, entre los 20 y 29 años, con tetraplejía incompleta, una clasificación ASIA D y la etiología más frecuente es la traumática.

La consistencia interna de la eSCIM III medida mediante el coeficiente Alfa de Cronbach fue muy alta (Alfa de Cronbach mayor de 0,9 en todas las evaluaciones). Esto indica que la eSCIM III tiene alta confiabilidad. Este análisis es fundamental cuando la escala, como lo es en nuestro caso, tiene subescalas. Otro aspecto importante es que no se mostró cambio eliminando alguno de los ítems de la escala, lo que indica que el cuestionario está correcto como se ha aplicado. En el caso de la eSCIM III todas sus subescalas contribuyen a la homogeneidad del instrumento.

La concordancia entre los dos evaluadores es muy alta para todas las subescalas e incluso se obtiene una concordancia absoluta (Índice de Kappa en 1) para la sub escala de movilidad en dormitorio y baño. La validación de la escala en Estados Unidos, por parte de Anderson y colaboradores muestra una concordancia entre los evaluadores entre el 65-99\%, ellos explican esta alta variabilidad entre evaluadores debido a la falta de un instructivo para la aplicación de la SCIM III, en nuestro caso consideramos que no es necesario, debido a la alta concordancia interevaluador obtenida. Las pocas diferencias encontradas entre los evaluadores pueden ser inherentes al examinador, al paciente evaluado o al instrumento por sí mismo. Además pueden ser debidos a la inexperiencia por parte de los evaluadores en aplicar la eSCIM III.

Para medir la validez externa se utiliza la correlación entre la MIF y la eSCIM III, en este caso se compararon ambas escalas al ingreso y al egreso de los pacientes. Se obtuvo en ambos casos un valor $\mathrm{p}<0,01$. La relación entre ambas escalas es alta debido a que las dos evalúan ítems como cuidado personal, manejo de esfínteres, traslados y movilidad.

En la sub escala de movilidad la SCIM III es más detallada, ya que se evalúa en el dormitorio, en el baño, y en exteriores a diferentes distancias. Además valora si el paciente logra o no realizar cambios de posición para la prevención de úlceras por presión. Otro aspecto importante de mencionar es que la MIF no valora la respiración, aspecto de suma relevancia en el proceso de rehabilitación de los pacientes con lesiones medulares.

Una limitación importante del presente estudio es haberlo realizado en un solo centro, lo que puede reducir la variabilidad de la muestra; esto podría mejorar con la inclusión de otros centros, como por ejemplo pacientes del Hospital del Trauma del Instituto Nacional de Seguros, lo que además permitiría la generalización de los resultados obtenidos.

\section{CONCLUSIONES}

Las características clínicas de la población estudiada son semejantes a los estudios previos tanto nacionales como internacionales de la población con lesiones medulares, lo cual señala la validez y aplicabilidad del estudio.

Se logra una adaptación aceptable y correcta de la eSCIM III, para la población de pacientes del Centro Nacional de Rehabilitación, a partir de los ajustes hechos propios de los usos del español de América.

La Medida de Independencia de la Médula Espinal versión III en español, es una herramienta válida y confiable para la evaluación funcional de los pacientes con lesiones medulares del Centro Nacional de Rehabilitación.

\section{BIBLIOGRAFÍA}

1. Nas K, Yazmalar L, Şah V, Aydın A \& Öneş K. (2015). Rehabilitation of spinal cord injuries. World J Orthop 18; 6(1): 8-16.

2. Zarco-Periñán M, Barrera-Chacón J, GarcíaObrero I, Méndez-Ferrer J, Alarcón L \& Echevarría-Ruiz de Vargas C. (2014). Development of the Spanish version of the Spinal 
Cord Independence Measure version III: cross-cultural adaptation and reliability and validity Study. Disability and Rehabilitation, 36(19): 1644-1651.

3. Catz A, Itzkovich M., Steinberg F., Tamir A., Ronen H. The Catz-Itzkovich "SCIM: a revised version of the Spinal Cord Independence Measure". Disability and Rehabilitation. 2001; Vol. 23, No. 6, 263- 268

4. Carvajal, A., Centeno, C., Watson, R., Martínez, M., \& Sanz Rubiales, Á. (2011). ¿Cómo validar un instrumento de medida de la salud? Anales Sis San Navarra, 34(1).

5. Anderson et al. (2011). United States (US) multi-center study to assess the validity and reliability of the Spinal Cord Independence Measure (SCIM III). Spinal Cord (2011) 49, 880-885

6. García Obrero, I., Echevarría Ruiz de Vargas, C., Sánchez Navarro, C., García Díaz, J., Polo Piñeiro, M., \& Rodríguez-Piñero Bravo-Ferrer, M. (1998). Escalas de valoración funcional en el paciente amputado. Rehabilitación, 32, 113-125.

7. Sánchez, R., \& Echeverry, J. (2004). Validación de escalas de medición en salud. Revista De Salud Pública, 6(3).

\section{CONFLICTOS DE INTERÉS}

Los autores declaran que no existen conflictos de interés. 